\title{
Tolosa-Hunt Syndrome: A Non-Classical Presentation of a Rare Cause of Unilateral Headache and Painful Ophthalmoplegia
}

\author{
Steven Douedia, d, Mark Awad ${ }^{\mathrm{b}}$, Daniel Shenouda ${ }^{\mathrm{a}}$, \\ Prinze Mack ${ }^{\mathrm{c}}$, Michael P. Carson ${ }^{\mathrm{a}}$
}

\begin{abstract}
Tolosa-Hunt syndrome is a rare condition involving the orbital and retro-orbital space. The typical symptoms are orbital pain, swelling, headache, palsies of the cranial nerves, and sensory loss in the distribution of the trigeminal nerve. Tolosa-Hunt syndrome relapses and remits with episodes separated by months to years. It is a diagnosis of exclusion after ruling out other causes of painful ophthalmoplegia and is treated with high-dose steroids. We present a case of a 43-year-old woman with a history of recurrent headaches and vision disturbances for the past 4 years presenting with worsening left-eye pain that radiated to the back of her head, swelling around the left eye, double vision, and a unilateral left-sided headache. Visual acuity was decreased in the left eye compared to the right and the patient reported left-eye pain when at extreme medial and lateral gaze. Erythrocyte sedimentation rate (ESR) was found to be elevated with all other laboratory testing within normal limits. Computed tomography (CT) scan of the orbits was unremarkable. Ophthalmology excluded other differential diagnoses and she was treated with intravenous methylprednisolone with significant improvement of symptoms. The triad of one or more episodes of unilateral orbital pain, paresis of one or more of the cranial nerves, and granulomas by magnetic resonance imaging (MRI) or biopsy is $95-100 \%$ sensitive at diagnosing the syndrome; however, our patient did not present with the "classic" triad. Initial treatment of Tolosa-Hunt is with high-dose steroids which as in our case lead to rapid and significant improvement of symptoms.
\end{abstract}

Keywords: Tolosa-Hunt syndrome; Painful ophthalmoplegia; Eye; Pain; Headache; Vision

Manuscript submitted February 25, 2020, accepted March 11, 2020

aDepartment of Medicine, Jersey Shore University Medical Center, Hackensack Meridian Health, Neptune, NJ 07753, USA

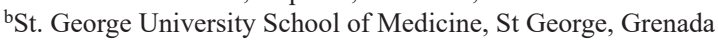

'Department of Ophthalmology, Jersey Shore University Medical Center, Hackensack Meridian Health, Neptune, NJ 07753, USA

${ }^{\mathrm{d} C}$ Corresponding Author: Steven Douedi, Department of Medicine, Jersey Shore University Medical Center, Hackensack Meridian Health, Neptune, NJ 07753, USA. Email: Steven.Douedi@hackensackmeridian.org

doi: https://doi.org/10.14740/jocmr4123

\section{Introduction}

Tolosa-Hunt syndrome, also called idiopathic orbital inflammation syndrome, is a rare neuro-immunological disorder that involves the contents of the orbital space and cavernous sinus. The cause is speculated to be an autoimmune reaction leading to an inflammatory process. The syndrome was first described in two papers, the first by Tolosa in 1954 presenting a middleaged man suffering from the disorder and the second in 1961 by Hunt et al who presented six patients with unilateral painful ophthalmoplegia [1]. We present a case of a unique presentation of Tolosa-Hunt syndrome in a middle-aged woman with rapid diagnosis and successful management of this rare cause of headache and ophthalmoplegia.

\section{Case Report}

A 43-year-old woman with a history of recurrent headaches and associated vision disturbances for the past 4 years presented to our emergency department with a 4-week history of progressive left-eye pain that radiated to the back of her head, swelling around the left eye, double vision, and a unilateral left-sided headache. There were no factors that worsened or improved her symptoms. She first developed this 4 years ago while living in another state. At that time, she was admitted to a hospital, given intravenous (IV) then oral steroids which helped suppress the pain and swelling, but she did not recall a specific diagnosis. Since that time, she has had three separate flare-ups, each resolving after steroid treatment, with the current episode being the most recent and severe. On physical exam she had left periorbital swelling and edema, 20/25 vision in her right eye and 20/30 in her left eye, and extra ocular muscles were intact, but she reported left-eye pain when at extreme medial and lateral gaze. She did not have temporal lobe tenderness on examination. There was normal muscle tone, range of motion, and sensation in all four extremities bilaterally. Laboratory results at admission were as follows: erythrocyte sedimentation rate (ESR) of $30 \mathrm{~mm} / \mathrm{h}$ and normal complete blood count, renal function, hepatic function, and transaminases. Computed tomography (CT) of the head without contrast, CT of the orbits with contrast (Fig. 1), CT angiogram of the head, and an electrocardiogram (EKG) were performed. All were normal aside 


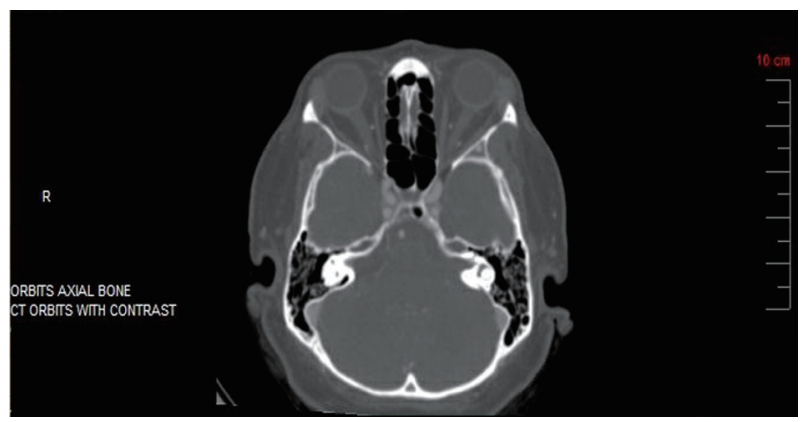

Figure 1. CT scan of orbits with contrast was unremarkable without orbital tissue swelling or masses. CT: computed tomography.

from the CT angiogram of the head which suggested a varix versus aneurysm involving the anterior cerebral arteries in the region of the inferior sagittal sinus (Fig. 2). Ophthalmology was consulted, excluded acute angle closure glaucoma, retinal disorders, diagnosed her with recurrent idiopathic orbital inflammation syndrome and possibly Tolosa-Hunt syndrome, and recommended treatment with methylprednisolone $80 \mathrm{mg}$ IV daily for 3 days. Within 24 h she noted a $50 \%$ reduction in pain and had complete resolution of her symptoms within 48 h. Magnetic resonance imaging (MRI) of the brain with and without contrast conducted the next day was normal and did not confirm the presence of a vascular anomaly suggested by the CT scan. At this point she was discharged on $60 \mathrm{mg}$ oral prednisone daily with a taper over the course of 2 weeks. One week after discharge she was seen at her ophthalmologist's office and remained asymptomatic.

\section{Discussion}

Tolosa-Hunt syndrome, or idiopathic orbital inflammation syndrome, is a rare neuro-immunological disorder affecting 1 - 2 per million people presenting with a classic triad of unilateral orbital pain, cranial nerve paresis, and granulomas on MRI or biopsy [2]. Tolosa-Hunt syndrome causes inflammation of the orbital space and cavernous sinus and can cause transient deficits of the third, fourth, and sixth cranial nerves, as well as parasympathetic fibers around the third cranial nerve $[2,3]$. It is diagnosed after excluding other causes of painful ophthalmoplegia such as neoplasms (meningiomas or schwannomas), multiple sclerosis, ischemic mononeuropathy, autoimmune disorders (Guillain-Barre syndrome, and myasthenia gravis), ophthalmoplegic migraine, tick bite paralysis, neuromuscular disorders, giant cell arteritis, malignancy, or vasculopathies including aneurysms of the posterior cerebral artery [4].

In the patient presented in this case, multiple sclerosis, neoplasms, and malignancies were ruled out using MRI and CT scans which were unremarkable. CT angiogram was performed and ruled out ischemia-related differential diagnoses. The patient only complained of left-eye pain and had an unremarkable musculoskeletal or sensory examination making Guillain-Barre syndrome and myasthenia gravis unlikely. The patient did not have temporal tenderness on palpation, vision

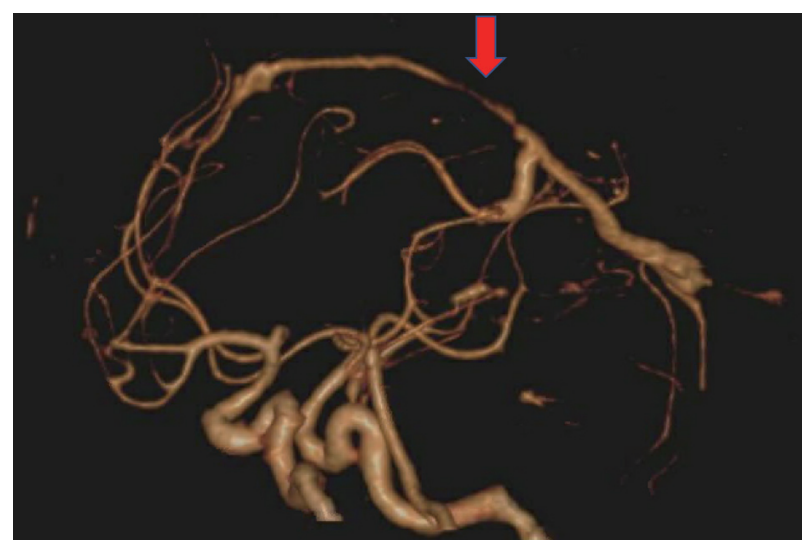

Figure 2. CT angiogram of head showing varix versus aneurysm involving the anterior cerebral arteries in the region of the inferior sagittal sinus (red arrow). CT: computed tomography.

loss, or fever making giant cell arteritis unlikely. While ophthalmoplegic migraine was high on our differential due to the patient presenting with recurrent headaches and can be the cause of unilateral pain and oculomotor cranial nerve palsy, the patient lacked a family history which is common for this type of migraine [5]. An elevated ESR along with a lack of aura and rapid improvement with steroids also helped rule out the diagnosis. A vital item in the differential diagnosis for Tolosa-Hunt syndrome is excluding a cavernous sinus thrombosis, which can occur due to septic or aseptic causes including polycythemia, sickle cell disease, vasculitis, pregnancy, dehydration, trauma, and intracranial surgery, none of which was found in our presented patient [5]. An additional cause of painful ophthalmoplegia is immunoglobulin G4 (IgG4)-associated disease, which is an idiopathic, multi-organ inflammatory state that can manifest as chronic, relapsing, sclerosing inflammation in virtually any organ system. Recent studies evaluating three cases of IgG4-associated disease have shown that histopathologic evaluation should be considered the gold standard in diagnosing the disorder which is typically treated with oral corticosteroids and other immunosuppressive drugs [6]. The patient presented in this case was only complaining of eye pain without systemic involvement making IgG4-associated disease unlikely.

The initial treatment of Tolosa-Hunt is high-dose steroids that are then tapered down upon remission [3]. Relapses separated by months to years are common. The triad of one or more episodes of unilateral orbital pain and paresis of one or more of the cranial nerves and granulomas by MRI or biopsy is 95$100 \%$ sensitive at diagnosing the syndrome, but the specificity is only $50 \%$ [4]. Without the classic triad, the term idiopathic orbital inflammation syndrome may be used, and the treatment is the same [4]. MRI usually shows enlargement or granulomatous changes in the cavernous sinus, superior orbital fissure, orbit affecting the optic nerve, and can reveal internal carotid artery narrowing $[5,7]$. However, the findings on MRI can be inconclusive and may be normal as described by Abdelghany et al and in our case [8]. Pain and paresis typically resolve within $72 \mathrm{~h}$ of corticosteroid treatment. The syndrome is classified as benign and self-limited, but the symptoms can be gru- 
eling for the patients affected.

For patients who present with eye pain and ocular nerve palsies, this case reviews the proper evaluation to collaborate with ophthalmology, exclude acute infection and malignancy, and once done consider the rare but treatable Tolosa-Hunt syndrome, also known as idiopathic orbital inflammation syndrome. It is vital that we exclude other causes for analogous symptoms as metastasis, lymphoma, aspergillosis, or even mucormycosis may share similar clinical and radiologic presentation with similar responses to steroids [1], and a missed diagnosis of these disorders can result in a fatal error. After excluding differentials, we were able to alleviate the patient's symptoms with corticosteroids, which are the mainstay of treatment [5].

\section{Conclusions}

Tolosa-Hunt syndrome is a rare, yet debilitating, disorder usually found to be a diagnosis of exclusion. The triad of one or more episodes of unilateral orbital pain, paresis of one or more of the cranial nerves, and granulomas by MRI or biopsy is $95-100 \%$ sensitive at diagnosing the syndrome. Despite the lack of the classic triad on presentation, her history and ability to exclude all other differential diagnoses led to her diagnosis and our management with high-dose steroids lead to marked improvement of symptoms and prevention of remissions thus far on 1-year follow-up. It is therefore important for clinicians to recognize Tolosa-Hunt syndrome in their differential of painful ophthalmoplegia and manage the condition promptly to alleviate worsening patient symptoms and improve care.

\section{Acknowledgments}

None to declare.

\section{Financial Disclosure}

None to declare.

\section{Conflict of Interest}

None to declare.

\section{Informed Consent}

The patient described in the case report had given informed consent for the case report to be published.

\section{Author Contributions}

Each author has individually been involved in and has made substantial contributions to case selection, case presentation, discussion, drafting manuscript and revision and final approval.

\section{Data Availability}

All data and information regarding this manuscript are present within the text.

\section{References}

1. Lueck CJ. Time to retire the Tolosa-Hunt syndrome? Pract Neurol. 2018;18(5):350-351.

2. Iaconetta G, Stella L, Esposito M, Cappabianca P. TolosaHunt syndrome extending in the cerebello-pontine angle. Cephalalgia. 2005;25(9):746-750.

3. Headache Classification Committee of the International Headache Society (IHS) The International Classification of Headache Disorders, 3rd edition. Cephalalgia. 2018;38(1):1-211.

4. Coppeto JR, Hoffman H. Tolosa-Hunt syndrome with proptosis mimicked by giant aneurysm of posterior cerebral artery. Arch Neurol. 1981;38(1):54-55.

5. Kline LB, Hoyt WF. The Tolosa-Hunt syndrome. J Neurol Neurosurg Psychiatry. 2001;71(5):577-582.

6. Lee CS, Harocopos GJ, Kraus CL, Lee AY, Van Stavern GP, Couch SM, Rao PK. IgG4-associated orbital and ocular inflammation. J Ophthalmic Inflamm Infect. 2015;5:15.

7. Schuknecht B, Sturm V, Huisman TA, Landau K. TolosaHunt syndrome: MR imaging features in 15 patients with 20 episodes of painful ophthalmoplegia. Eur J Radiol. 2009;69(3):445-453.

8. Abdelghany M, Orozco D, Fink W, Begley C. Probable Tolosa-Hunt syndrome with a normal MRI. Cephalalgia. 2015;35(5):449-452 . 